Proc. Indian Acad. Sci. (Earth Planet. Sci.), Vol. 95, No. 3, November 1986, pp. 321-330.

(C) Printed in India.

\title{
Computation of synthetic seismograms for plane layered earth models including absorption and dispersion phenomena
}

\author{
K N S YADAV and AVADH RAM \\ Department of Geophysics, Banaras Hindu University, Varanasi 221 005, India
}

MS received 30 May 1983; revised 15 November 1984

\begin{abstract}
A computer program package has been written in FORTRAN-IV language and tested successfully on an ICL 1904 S computer. This program enables one to compute synthetic seismograms for layered earth models. The provision for studying the effect of absorption and dispersion of seismic waves has been made with Subroutines. The present program utilizes eight Subroutines and requires about $35 \mathrm{~K}$ core memory. A set of examples are illustrated for absorption and dispersion models. An exponential decay of amplitude has been used for the absorption model. This method is based on the plane wave propagation in a flat-layered earth system. Normal incident P-waves are used to eliminate the effect of other phases. Change in shape of reflected waves is observed in absorption model due to damping of energy of higher frequencies. Lack of resolution is found between closely spaced reflections at higher frequencies. The effect of dispersion on seismic waves decreases the time of primary reflections as well as amplitudes of the seismic waves.
\end{abstract}

Keywords. Synthetic seismograms; computer programs; absorption; dispersion.

\section{Introduction}

The problem of wave propagation from a seismic source in a layered medium can be addressed either through ray theory methods or by using wave theory approach. In the latter approach a direct numerical evaluation of integral representing the frequency domain solution is required. In this domain, approximate computational methods have been developed by Pinnery (1965), Fuchs (1968), Fuchs and Muller (1971), Bouchon and Aki (1977) and Bouchon (1979) and have introduced the concept of a discretized wave field to obtain the solution in the near field of the source. In the past, a number of techniques for computation of synthetic seismograms have been developed (Wuenschel 1960; Trorey 1962; Claerbout 1968; Trietal and Robinson 1966; Robinson 1968; Robinson and Trietal 1977; Khattri et al 1978; Kennet and Kerry 1979; Kennet 1980; Kennet and Illingworth 1981). They have considered different phenomena associated with the earth's crust. In most of these cases, effects of seismic energy loss have been ignored, but Trorey (1962) introduced an approach which allowed the inclusion of attenuation. Khattri et al (1978) considered the frequency-dependent absorption and velocity free from dispersion. Acoustic wave propagation in porous media has been studied by several workers (Biot 1962 a, b; Deresiewicz and Rice 1962, 1964; Deresiewicz and Levv 1967; Deresiewicz 1974 and Burridge and Vargas 1979). Deresicwicz (1974) showed that the presence of liquid in the pores causes a decrease in the phase velocity and motion is attenuated. More recently, there has been considerable interest for problems of absorption and dispersion. Ganley (1981) considered such 
problems and gave a method to generate synthetic seismograms which incorporate these effects. His technique can be used to produce normal incidence, plane wave seismograms for flat-layered earth models. It is essentially a modification of communication theory approach to synthetic seismograms.

In this article, we have developed a set of computer programs for the computation of synthetic seismograms based on the theory proposed by Ganley (1981) as it is straightforward and includes the effect of absorption and dispersion. These seismograms are useful in the study of effect of changes in lithology. This program contains eight Subroutines which are listed with their functions in table 1. Input and output of the Subroutines are given in table 2. This program requires $35 \mathrm{~K}$ core memory for the type of cases taken up in the present study. The program has been tested on an ICL 1904 S computer. We have presented the results of an assumed model which contains sandy oil, gas and water overlain and underlain by shale to form a trap. Bed rock is assumed to be sandstone as a lower half space. In computation, an unit spike is taken as a signal and source-receivers configuration is assumed at the surface.

\section{Computational procedure}

Our programming formulation has been taken from the method given by Ganley (1981). The computation of synthetic seismograms is based on the well-known absorption model of exponential decay of amplitude with distance which is given by;

$$
A=A_{0} \exp (-\alpha z)
$$

where $A_{0}$ is the initial amplitude and $A$, the amplitude at depth $z, \alpha$ the absorption coefficient which is frequency-dependent, usually written as

$$
\alpha=|w| / 2 c Q
$$

where $|w|$ is the absolute angular frequency, $c$ the phase velocity and $Q$ the quality factor.

\begin{tabular}{|c|c|}
\hline Subroutines & Function \\
\hline $\begin{array}{l}\text { SYNTH } 1 \\
\text { SYNTH } 2\end{array}$ & $\begin{array}{l}\text { Calculates attenuation, velocity and quality factor as a function of frequency. } \\
\text { Makes velocity complex function of frequency and computes reflection and } \\
\text { transmission coefficients, using output of SYNTH } 1 \text {. }\end{array}$ \\
\hline WIDSYNTH & $\begin{array}{l}\text { Computes reflection and transmission coefficients without considering the } \\
\text { frequency effect. }\end{array}$ \\
\hline SYNTH 3 & $\begin{array}{l}\text { Computes spectrum of upgoing and downgoing waves using output from } \\
\text { SYNTH 2/WIDSYNTH. }\end{array}$ \\
\hline SYNTH 4 & $\begin{array}{l}\text { Computes synthetic seismograms on the basis of spectrum obtained from } \\
\text { SYNTH 3. }\end{array}$ \\
\hline REFL & Provides complex conjugate to use in NLOGN Subroutine. \\
\hline NLOGN & Performs the Fourier transformation. \\
\hline MAXSN & Finds the maximum value to normalize the desired output. \\
\hline
\end{tabular}

Table 1. List of Subroutines required for the computation of synthetic seismograms. 
Table 2. Variables used in different Subroutines.

\begin{tabular}{|c|c|c|}
\hline \multirow[b]{2}{*}{ Subroutines } & \multicolumn{2}{|c|}{ Variables } \\
\hline & Input & Output \\
\hline SYNTH 1 & $\begin{array}{l}\text { W=angular frequency } \\
N O M=\text { number of points } \\
C=\text { phase velocity } \\
Q=\text { quality factor } \\
\mathrm{NL}=\text { number of layers in a model }\end{array}$ & $\begin{array}{l}A L P=\text { attenuation coefficient } \\
C W=\text { frequency-dependent } \\
\text { velocity } \\
Q D=\text { frequency-dependent } Q \\
l\end{array}$ \\
\hline SYNTH 2 & $\begin{array}{l}\mathrm{C}, \mathrm{CW}, \mathrm{Q}, \mathrm{QD}, \mathrm{NOM}, \mathrm{NL}, \\
\mathrm{W}, \mathrm{DEN}=\text { density of layer }\end{array}$ & $\begin{array}{l}\text { Complex } \\
\text { VC=velocity } \\
R C=\text { reflection coefficient } \\
T C=\text { transmission coefficient }\end{array}$ \\
\hline WIDSYNTH & $\mathrm{C}, \mathrm{Q}, \mathrm{DEN}, \mathrm{NOM}, \mathrm{NL}$ & Complex VC, RC,TC \\
\hline SYNTH 3 & $\begin{array}{l}\mathrm{RC}, \mathrm{TC}, \mathrm{ALP}, \mathrm{W}, \mathrm{NOM}, \mathrm{NL} \text {, } \\
\mathrm{TH}=\text { thickness of layer } \\
\mathrm{TR}=\text { travel time in layer }\end{array}$ & $\mathrm{U}=$ spectrum of the upgoing wave \\
\hline SYNTH 4 & $\mathrm{U}, \mathrm{NOM}, \mathrm{NL}, \mathrm{F}=\underset{\text { frequency. }}{\text { linear }}$ & $\begin{array}{l}\text { AMP }=\text { amplitude } \\
\text { PHA }=\text { phase }\end{array}$ \\
\hline REFL & $\mathrm{U}, \mathrm{NOM}$ & $\begin{array}{l}\mathrm{RF}(=\mathrm{U} \text { with its complex } \\
\text { conjugate })\end{array}$ \\
\hline NLOGN & NT=integer number and RF & AMP \\
\hline MAXSN & $\mathrm{NN}=2 \times \mathrm{NOM}, \mathrm{AMP}, \mathrm{PHA}$ & $\begin{array}{l}\text { XMAX =maximum value of } \\
\text { amplitude/phase }\end{array}$ \\
\hline
\end{tabular}

We have used Futterman's (1962) theory as it provides an excellent model of attenuation and dispersion that is in good agreement with experimental data. It is based on the principle of superposition and linearity of absorption coefficient as a function of frequency. However, some other absorption-dispersion pair could also be used.

Using the Futterman's (1962) absorption-dispersion pair, the absorption coefficient $\alpha$ can be written as,

$$
\alpha(w)=w / 2 c Q^{\prime}=w / 2 c_{0} Q_{0} .
$$

The frequency-dependent phase velocity is given by

$$
c(\omega)=c_{0}\left[1-\frac{1}{\pi Q_{0}} \ln \frac{\nu \omega}{\omega_{0}}\right]^{-1}
$$

and

$$
Q^{\prime}(w)=Q_{0}\left[1-\frac{1}{\pi Q_{0}} \ln \frac{\nu \omega}{\omega_{0}}\right]
$$

where $\ln \nu=0.5772157 . \omega_{0}$ is the arbitrary low cut-off frequency, usually chosen much less than $\Delta w$ (the angular frequency spacing) and $Q^{\prime}$ is the modified quality factor. 
At $w_{0}$ frequency (approximately zero), there is no dispersion and the phase velocity approaches $c_{0}$, quality factor $Q_{0}$, as $\omega \rightarrow \omega_{0}$. The reflection and transmission coefficient will be real at that frequency. However, the principle of causality is violated if $Q$ depends on $w$ linearly as it tends to infinity. For non-zero frequency, velocity is made a complex function. The real part of velocity is given by

$$
V_{r}=c / 2\left[1+\left(1+1 / Q^{2}\right)^{-1 / 2}\right]
$$

and the complex velocity is

$$
V(w)=V_{r}\left[1+\frac{i \operatorname{sgn}(w)}{2 Q^{\prime}}\right]
$$

where $\operatorname{sgn}(w)=1$ for $w>0$ and -1 for $w<0 . V_{r}$ is the real velocity which is constant for a particular layer and $V(w)$ is the complex velocity which is not constant for the layer but it varies with frequency and quality factor of that layer. For dispersive case complex velocity is used to calculate the reflection and transmission coefficients as follows:

$$
R=\left(\rho_{2} V_{2}-\rho_{1} V_{1}\right) /\left(\rho_{1} V_{1}+\rho_{2} V_{2}\right)
$$

and

$$
T=\left(2 \rho_{2} V_{2}\right) /\left(\rho_{1} V_{1}+\rho_{2} V_{2}\right),
$$

where $\rho$ is the density of the layer. If $U_{i}$ and $D_{i}$ are the spectrums of the upgoing wave and downgoing waves respectively in the $i$ th layer, these are related as

and

$$
D_{i}=\frac{\exp \left(\alpha d_{i}\right) \exp \left(i \omega d_{i} / c_{i}\right)}{T_{i}}\left(D_{i+1}+R_{i} U_{i+1}\right)
$$

$$
U_{i}=\frac{\exp \left(-\alpha d_{i}\right) \exp \left(-i \omega d_{i} / c_{i}\right)}{T_{i}}\left(R_{i} D_{i+1}+U_{i+1}\right)
$$

where $d_{i}$ is the thickness of the $i$ th layer. The ratio of the spectrums of upgoing and downgoing waves are given by

$$
Y_{i}=U_{i} / D_{i}=\exp \left(-2 \alpha d_{i}\right) \exp \left(-2 i \omega d_{i} / c_{i}\right)\left(\frac{R_{i}+Y_{i+1}}{1+R_{i} Y_{i+1}}\right)
$$

For an $n$-layer model $U_{n+1}=0$ and therefore $Y_{n+1}=0$. Equation (12) can be successively applied from the bottom layer to the surface until $Y_{1}$ is determined.

If $R_{0}$ is the reflection coefficient of the surface as seen from above, then $-R_{0}$ will be the reflection coefficient of the surface for upgoing wave in the first layer, and

$$
D_{1}(w)=1-R_{0} U_{1}(w) \text {. }
$$

With knowledge of $U_{1}(w)$ and $D_{1}(w)$, the Fourier transform of synthetic seismogram, $X(w)$ at the surface is given by

$$
\begin{aligned}
X(w) & =U_{1}(w)+D_{1}(w), \\
& =\left(1-R_{0}\right) U_{1}(w)+1 .
\end{aligned}
$$


Therefore, given an $n$-layer model, it is possible to calculate the Fourier transform (FT) of the surface synthetic seismogram for an initial downgoing spike at the surface. This FT can then be multiplied by the FT of the wavelet if we wish to use a wavelet different from a spike as the input wavelet.

We have developed computer programs based on the above equations. Part-wise calculations are performed using different Subroutines (table 1). Subroutine SYNTH 1 calculates the attenuation coefficient, frequency-dependent velocity and modified quality factor depending on equations (3), (4) and (5). Subroutine SYNTH 2 first makes the velocity as a complex function, then reflection and transmission coefficients are calculated for dispersive case using (6) to (9).

We have also computed synthetic seismograms without the effect of dispersion. For this purpose, a separate Subroutine WIDSYNTH is developed. After computations of reflection and transmission coefficients of different layers, we have calculated the ratio of the spectrum of the upgoing and downgoing waves with the help of Subroutine SYNTH 3, which is based on (12). Surface layer synthetic seismograms in frequency domain. are computed using (13) and (14). This is performed in Subroutine SYNTH 4. Time domain synthetic seismograms are computed using Subroutine NLOGN. Subroutine REFL is used to find out the complex conjugate of the frequency function, calculated only at positive frequencies. Maximum values of the amplitude and phases are obtained with Subroutine MAXSN, which are used to normalize the output.

\section{Examples of synthetic seismograms}

In this exercise a number of theoretical data were assumed for different layers to check the validity of the programs in the close proximity of the real field situation. We used a model whose relevant parameters are given in table 3 . Velocity, density, thickness and quality factors have been used as an input data. $Q$ values and phase velocities given in table 3 are assumed at zero frequency. Lower and upper cut-off frequencies are set to be $0.001 \mathrm{~Hz}$ and $125.0 \mathrm{~Hz}$ respectively. Since the amplitude for negative values of frequency $(w)$ is the complex conjugate of the corresponding positive values, computations are made for positive frequencies at 65 points including $w=0$. The interval between consecutive samples in the frequency domain is $1.953 \mathrm{~Hz}(=125 / 64)$. Further the number of samples obtained at Nyquist interval of $4 \mathrm{msec}$ is required to be an integral power of 2 in order to apply Fourier

Table 3. Various parameters of the model used in computation of synthetic seismograms.

\begin{tabular}{lcccc}
\hline Lithology & $\begin{array}{c}\text { Velocity } \\
(\mathrm{m} / \mathrm{sec})\end{array}$ & $\begin{array}{c}\text { Density } \\
(\mathrm{gram} / \mathrm{cc})\end{array}$ & $\begin{array}{c}\text { Thickness } \\
\text { (meter) }\end{array}$ & $\begin{array}{c}\text { Quality } \\
\text { factor }\end{array}$ \\
\hline Shale & 2700 & $2 \cdot 23$ & 200 & $30 \cdot 9$ \\
gas-sand & 1700 & $2 \cdot 05$ & 25 & $21 \cdot 4$ \\
oil-sand & 3040 & $2 \cdot 29$ & 30 & $56 \cdot 0$ \\
water-sand & 3536 & $2 \cdot 32$ & 58 & $64 \cdot 8$ \\
Shale & 3049 & $2 \cdot 32$ & 105 & $39 \cdot 2$ \\
sandstone & 4090 & $2 \cdot 45$ & - & $75 \cdot 4$ \\
\hline
\end{tabular}


Transform in our case for time domain calculation. The number of samples in both frequency and time domains should be equal. Thus it yields $128\left(=2^{7}\right)$ samples in both the domains.

\subsection{Non-dispersive model}

In this model two cases have been considered, viz (i) without absorption and (ii) with absorption. Figure 1a shows the amplitude spectrum of a non-dispersive model without absorption. The maximum amplitude of oscillations has been found in the frequency range greater than $50 \mathrm{~Hz}$. In this spectrum, constructive and destructive interferences are recognized by maxima and minima respectively. Figure $1 b$ illustrates the amplitude spectrum of the non-dispersive dissipative model. It is clear from this case that amplitudes are highly attenuated at frequencies greater than $60 \mathrm{~Hz}$. At lower frequencies, the attenuation is not very much pronounced. Figures $2 \mathrm{a}$ and $2 \mathrm{~b}$ show the corresponding phase spectra of the model. It is observed that phases vary between $\pm 1 \cdot 0$ radian. The oscillations in phase spectra in the dissipative case show high attenuation of phase in the higher frequency range greater than $60 \mathrm{~Hz}$, which may be attributed to attenuation of the wave-form within this domain.

The corresponding synthetic seismograms have been computed from the frequency spectra using inverse Fourier transform and are shown in figures $3 \mathrm{~b}$ and 3c. Time section is also illustrated against two-way travel time in msec (figure 3a).

AMPLITUDE SPECTRUM
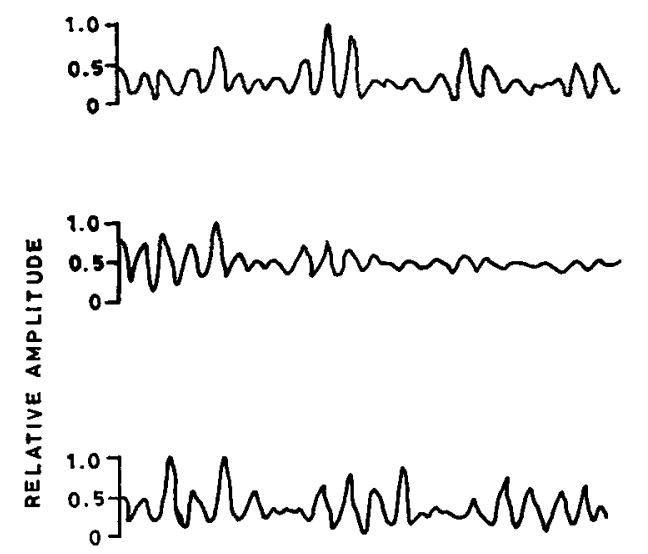

(B)

(C)

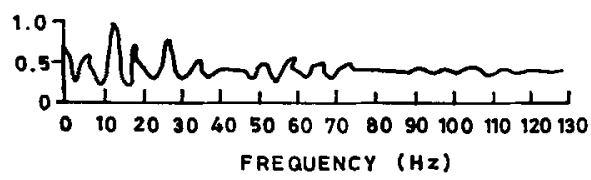

(0)

Figure 1. Amptitude spectra of different models (a) and (b). Non-dispersive models without and with absorption. (c) and (d). Dispersise models without and with absorption 


\section{PHASE SPECTRUM}
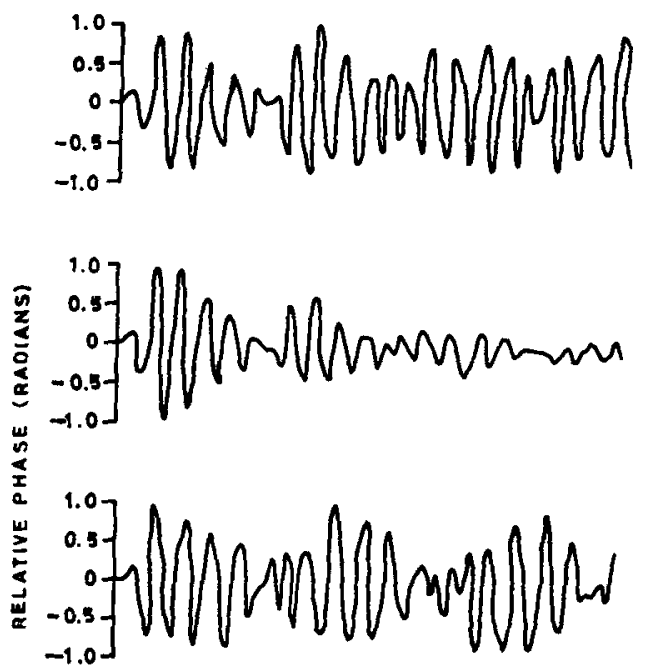

(B)

(c)

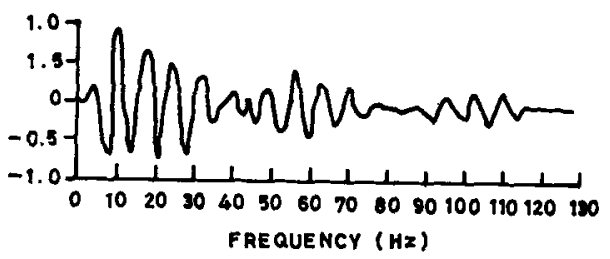

(D)

Figure 2. Phase spectra of various models. (a) and (b). Non-dispersive models without and with attenuation. (c) and (d). Dispersive models without and with attenuation.

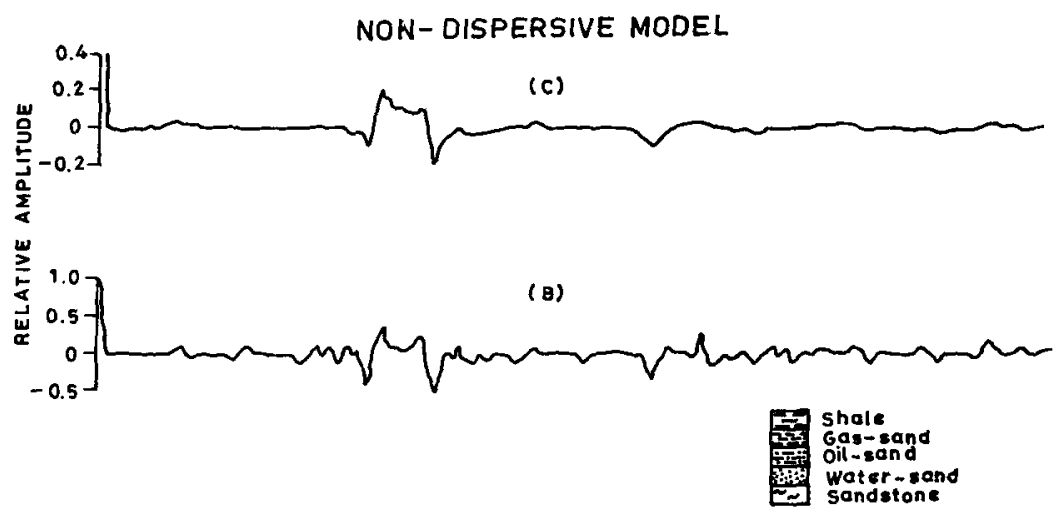

(A)

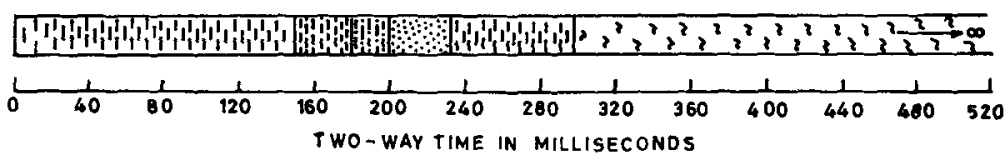

Figure 3. Synthetic seismograms along with geological section for non-dispersive model. (a) Lithological section against two-way time in msec. (b) and (c). Synthetic seismograms without and with absorption. 
Figures $3 \mathrm{~b}$ and $3 \mathrm{c}$ represent loss-less and dissipative seismograms for a nondispersive case. It is clear from these diagrams that boundaries are well marked by reflection amplitudes. High amplitudes are observed at shalegas and gas-oil boundaries. However, amplitudes are attenuated much for the dissipative case as shown in figure $3 \mathrm{c}$.

\subsection{Dispersive model}

In this model, effects of dispersion (i) with absorption and (ii) without absorption are considered separately as in the previous model. Figures $1 \mathrm{c}$ and $1 \mathrm{~d}$ illustrate the nonabsorptive and absorptive cases of amplitude spectra. In this case, maximum amplitude of oscillations is observed in the frequency range less than $30 \mathrm{~Hz}$. The period of oscillations is approximately similar as in the case of nondispersive model, but the maxima and minima positions are displaced which illustrates interference patterns at another frequencies in comparison to the previous model. In the absorption model (figure 1d) attenuation is more pronounced at higher frequencies greater than $70 \mathrm{~Hz}$ than that of the dissipative case of nondispersive model. Figures $2 \mathrm{c}$ and $2 \mathrm{~d}$ show the corresponding phase spectra of loss-less and dissipative models. Phase variations in these cases are found between +1.0 and -0.8 radian. Phases are highly attenuated for dissipative model at higher frequencies range greater than $60 \mathrm{~Hz}$. Phase variation in the dispersive loss-less model (figure 2c) is low in comparison to nondispersive loss-less model (figure $2 \mathrm{a}$ ) in two frequency ranges around $80 \mathrm{~Hz}$ and $120 \mathrm{~Hz}$.

Figure 4 shows the synthetic seismograms of the dispersive model. Time section in this case is identical as was considered for nondispersive model to compare arrivals of primary reflections (figure $4 \mathrm{a}$ ). In this case, reflection positions are found to be displaced showing the effect of dispersion. The pattern of seismograms is similar to nondispersive case with decreased amplitudes for both the nondissipative and dissipative cases (figures $4 b$ and $4 c$ ). It is observed that dispersion effect produces $15 \mathrm{msec}$ time difference in primary reflections in comparison to nondispersive case.

\subsection{Discussion and conclusion}

The concept of utilizing the sedimentation models to study the possibilities of determining stratigraphic variations and locating stratigraphic traps through the study of synthetic seismograms has been presented. Stratigraphic traps are important for the accumulation of petroleum, resulting from the change in lithology and environment of deposition.

Amplitude spectra of nondispersive and dispersive models show identical period of oscillations but maximum amplitude positions are observed at different frequencies, viz $52 \mathrm{~Hz}$ in nondispersive case and $26 \mathrm{~Hz}$ in dispersive case. Maxima and minima positions are not coincident over the entire frequency range except $50-60 \mathrm{~Hz}$. The exception of this frequency range may be explained by the fact that the maximum contribution to reflected signals, which arise from shale-gas and oil-gas boundaries does not contain low frequency component and thus passes 
DISPERSIVE MOOEL

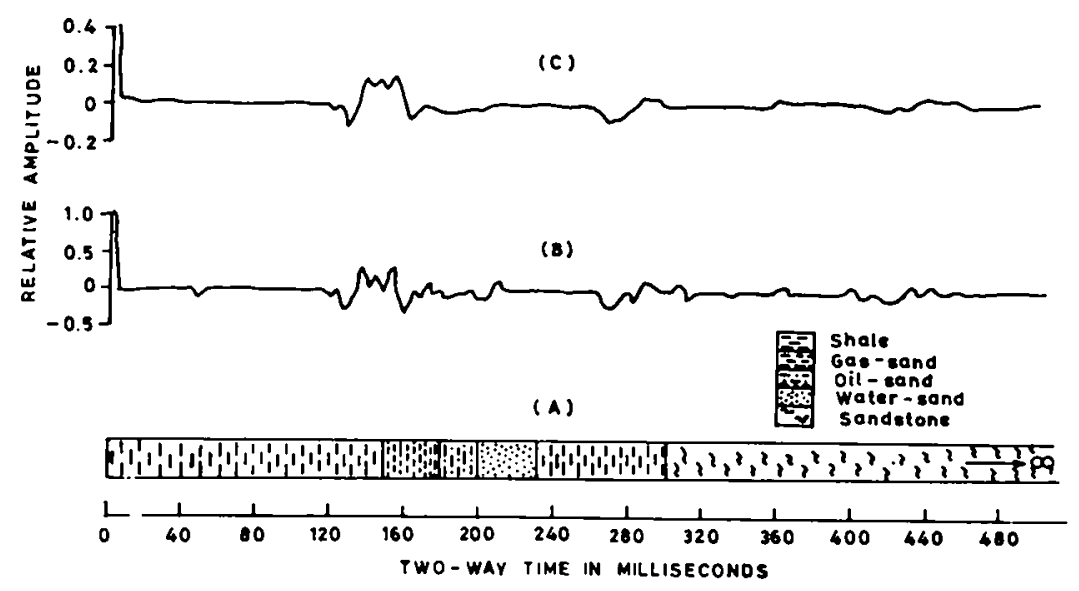

Figure 4. Synthetic seismograms alongwith geological section for the dispersive model. (a). Lithological section against two-way time in msec. (b) and (c). Synthetic seismograms without and with absorption.

through virtually unaffected. The closest similarity between the two spectra is observed at frequencies less than $8 \mathrm{~Hz}$. It is clear from figure 1 that absorption is more pronounced in the dispersive case as compared to the nondispersive model. Figure 2 shows the variation of phases for different models. In the case of loss-less dispersive/nondispersive model, phase variations are approximately between $\pm 1 \cdot 0$ radian. Change in phases for both the dissipative models has been observed at higher frequency greater than $60 \mathrm{~Hz}$, which may be attributed to the absorption effect. However, the cause for the change in phase in dispersive model (figure 2c) around $80 \mathrm{~Hz}$ and $120 \mathrm{~Hz}$ is not precisely known.

Synthetic seismograms (figures 3 and 4 ) show similarity in the oscillations. It is found that the presence of gas produces high amplitude reflections in both the models. The amplitude in dispersive case is less in comparison to nondispersive case. The effect of dispersion is to decrease the arrival time of primary reflection and wave amplitudes. Absorption effect in both cases creates change in shape of reflections by damping the energy of higher frequencies. It also broadens the pulses and lack of resolution is observed between closely-spaced reflections at higher frequencies. It is clear from the present discussion that the frequency domain calculation is useful to evaluate the spectral response of elastic and dissipative earth models.

The present computer program package enables one to study the effect of changes in lithological characters by generating the synthetic seismograms for a plane-layered earth system. The required core memory is suitable for moderatelysized computers. These programs are stored in the disk file for ready use whenever necessary. We are now trying to study the effects of oblique incident wave on the interfaces using modified Subroutines. Attempts are being made to study the effects of scattering and diffraction on the seismic waves for different geological models. The results of these investigations would be published elsewhere. 


\section{Acknowledgements}

The authors are thankful to the Director, KDM IPE, Oil and Natural Gas

Commission, Dehradun for financial assistance.

\section{References}

Biot. M A 1962a J. Appl. Phys. 331482

Biot M A 1962b J. Accoust. Soc. Am. 341256

Bouchon M 1979 J. Geophys. Res. 843609

Bouchon M and Aki K 1977 Bull. Seism. Soc. Am. 67259

Claerbout J F 1968 Geophysics 33264

Burridge R and Vargas C A 1979 Geophys. J. R. Astron. Soc. 5861

Deresiewicz H 1974 Bull. Seismol. Soc. Am. 641901

Deresiewicz H and Levy A 1967 Bull. Seismol. Soc. Am. 57381

Deresiewicz H and Rice J T 1962 Bull. Seismol. Soc. Am. 52595

Deresiewicz H and Rice J T 1964 Bull. Seismol. Soc. Am. 54409

Fuchs K 1968 J. Phys. Earth 1627

Fuchs K and Muller G 1971 Geophys. J. R. Astron. Soc. 28417

Futterman W I 1962 J. Geophys. Res. 695279

Ganley D C 1981 Geophysics 461100

Kennet B L N 1980 Geophys. J. R. Astron. Soc. 611

Kennet B L N and Illingworth M R 1981 Geophys. J. R. Astron. Soc. 66633

Kennet B L N and Kerry N J 1979 Geophys. J. R. Astron. Soc. 57557

Khattri K, Gaur V K, Mittal R and Tandon A K 1978 Geoexploration 16185

Pinnery R A 1965 J. Geophys. Res. 705107

Robinson E A 1968 Geophysics 33521

Robinson E A and Trietal S 1977 Geophys. Prosp. 25434

Trietal S and Robinson E A 1966 Geophysics 3117

Trorey A W 1962 Geophysics 27766

Wuenschel P C 1960 Geophysics 25106 